\title{
TOXOPLASMOSE OCULAR EM CÃES JOVENS \\ INOCULADOS COM Toxoplasma gondii ${ }^{1}$
}

\author{
OCULAR TOXOPLASMOSIS IN YOUNG DOGS \\ INOCULATED WITH Toxoplasma gondii
}

\begin{abstract}
Cláudia Bonini de Abreu ${ }^{2}$ Italmar Teodorico Navarro ${ }^{3}$ Antônio Carlos Farias dos Reis ${ }^{3}$ Mírian Siliane Batista de Souza ${ }^{4}$ Roberto Machado ${ }^{5}$ Elizabete Regina Marangoni Marana ${ }^{6}$ Leonardo Begale Prudêncio ${ }^{7}$ Marcos Rodrigues Mattos ${ }^{7}$ Vinícius Suehiro Tsutsui ${ }^{7}$
\end{abstract}

RESUMO

A toxoplasmose é uma doença zoonótica de distribuição mundial que pode infectar uma grande variedade de espécies animais e o homem. No presente trabalho, foram avaliados nove cães jovens inoculados experimentalmente com três cepas diferentes de $\boldsymbol{T}$. gondii em que se observaram através de microscopia direta e indireta, alterações oftálmicas em sete deles, confirmadas através de retinografia e histopatologia. Com os resultados obtidos, observou-se que as alterações oftálmicas na toxoplasmose adquirida nos cães apareceram já ao término da terceira semana pós-inoculação, mostrando a precocidadedas alterações. No exame direto do olho, sete cães apresentaram áreas de hiperrefletividade. Alterações como papiledema, exsudato peripapilar e diminuição da pigmentação do tapetum foram observadas em três cães submetidos à retinografia. $O$ exame histopatológico revelou a presença de discreto infiltrado mononuclear na córnea na região subepitelial em estroma conjuntivo em nível do ângulo da íris, congestão vascular da coróide e edema sub-retiniano em oito cães. Nossos resultados permitem concluir que a toxoplasmose ocular adquirida nos cães é tão significativa quanto na espécie humana. Assim, estudos devem ser aprofundados nesta linha de pesquisa para reforçar os achados.

Palavras-chave: toxoplasmose, Toxoplasma gondii toxoplasmose ocular, cães.

SUMMARY

Toxoplasmosis is a zoonotic disease of worldwide distribution that can infect a great variety of animal species and man. In the present study nine young dogs inoculated with three different samples of Toxoplasma gondii were evaluated. Seven of them showed ophthalmic alterations by means of direct and indirect microscopy, photomicrograph and histopathology. From the results obtained it was noted that ophthalmic alterations were already present at the end of the third week after inoculation, showing the precocity of such alterations. In the direct examination of the eyes, seven dogs presented increased reflective areas. Alterations such as papilledema, peripapillary exsudate and tapetum pigmentation were noted in three dogs submitted to retinograph. The histopathology showed focal mononuclear infiltrate of choroid, congestion of chorioretinal vessels an subretinal edema in eight dogs. Our results allow to conclude that acquired ocular toxoplasmosis in the dogs is as significant as in man and in doing so other studies must be undertaken.

Key words: toxoplasmosis, Toxoplasma gondii, ocular toxoplasmosis, dogs.

\section{INTRODUÇÃO}

O Toxoplasma gondii está amplamente distribuído no mundo (DUBEY, 1993) e pode infectar todos os animais de sangue quente (BANETH $\boldsymbol{e t}$ al., 1996). Por isso, é considerada uma protozoonose, seja para os homens, seja para os animais, de grande interesse, tendo em vista as sérias consequiências da infecção (DUBEY et al., 1995 ).

\footnotetext{
${ }^{1}$ Projeto financiado pela Centro de Pós-graduação da Universidade Estadual de Londrina (UEL), Londrina, PR. CAPES, CNPq. ${ }^{2}$ Professor, Universidade de Marília, Marília, SP, Mestrando de Sanidade Animal, Centro de Ciências Agrárias (CCA), Departamento de Medicina Veterinária Preventiva (DMVP), UEL.

${ }^{3}$ Professor do DMVP, UEL, CP 6001, 86050-970, Londrina, PR. E-mail: italmar@uel.br. Autor para correspôndencia.

${ }^{4}$ Professor, Departamento de Clínica Veterinária, UEL.

${ }^{5}$ Oftalmologista, Departamento de Clínica Médica, Centro de Ciências da Saúde, UEL.

${ }^{6}$ Médico Veterinário, Mestre em Sanidade Animal, CCA, DMVP. Bolsista CNPq.
} 
Sociedades onde existe alto consumo de carnes cruas ou mal cozidas e regiões com falta de saneamento apresentam taxas mais altas de infecção (AMATO NETO et al., 1995). Entre os poucos trabalhos de inoculação experimental em cães encontrados na literatura, destaca-se o de FIALHO (1953), que estudou a toxoplasmose experimental no cão e destacou como principal alteração lesões oculares. PIPER et al. (1970) descreveu retinite, coroidite e presença de pseudocisto de $T$. gondii no epitélio ciliar de cão portador de infecção toxoplásmica. A falta de informações na literatura sobre alterações do sistema oftálmico do cão por este protozoário tem levado a inferir os achados da literatura no homem. Estudos subseqüentes dos tecidos oculares de crianças revelaram a existência de $\boldsymbol{T}$. gondii exclusivamente na retina, porém com severa inflamação da coróide subjacente (HOLLAND et al., 1995). As lesões crônicas ou recidivantes apresentam comprometimento vítreo mais intenso, que parece estar relacionado com alterações imunológicas que necessitam de doses altas de corticóides para serem removidas (ABREU et al., 1987).

A forma adquirida da toxoplasmose ocular em humanos é a mais freqüente, segundo SILVEIRA (1997), ao contrário das citações de AMATO NETO et al. (1995) que indicam a forma congênita como a mais freqüente. Os inúmeros cães com problemas oftálmicos em ambulatórios e a falta de informações na literatura levaram à proposição do presente estudo, que teve como objetivo estudar possíveis alterações oftálmicas em cães jovens inoculados com diferentes amostras de $T$. gondii.

\section{MATERIAL E MÉTODOS}

No presente trabalho, foram utilizadas três cepas de $\boldsymbol{T}$. gondii, com características particulares quanto a patogenia e à patogenicidade em camundongos. A escolha das cepas foi determinada pela falta de informações, na literatura, quanto aos mecanismos que levam a provocar lesões oculares ou tropismo pelo olho. As cepas são denominadas de $\mathrm{RH}$, isolada de ser humano com característica à resposta aguda, e a não cronificação, AS-28, isolada de camundongo, e $\mathrm{P}$, isolada de cão, ambas com característica à cronificação formando cistos $\mathrm{e}$ oocistos quando inoculadas em gatos. As cepas RH e AS-28 foram mantidas no Laboratório de Protozoologia do Departamento de Medicina Veterinária Preventiva, da Universidade Estadual de Londrina (UEL), e a cepa $\mathrm{P}$ foi mantida junto ao Departamento de Patologia Veterinária, da Faculdade de Ciências Agrárias e Veterinárias de
Jaboticabal (FCAVJ - UNESP), através de sucessivas passagens em camundongos albinos.

Foram selecionados 12 cães, com idade aproximada de 30 dias, sorologicamente negativos pela Reação de Imunofluorescência Indireta (RIFI) para T. gondii (CAMARGO, 1964). Esses animais foram mantidos em jaulas individuais, durante todo o período de adaptação (4 meses), Receberam vacinas, anti-helmínticos, água à vontade e ração comercial para filhotes. Foram ainda acompanhados com exames clínicos completos e laboratoriais.

Cada um dos dois grupos, compostos de três animais, foi inoculado, respectivamente, com $5 \mathrm{~m} \ell$ (contendo $1,8 \times 10^{4}$ oocistos $/ \mathrm{m} \ell$ ) das cepas $\mathrm{P}$ e $5 \mathrm{~m} \ell\left(1,6 \times 10^{4}\right.$ oocistos $\left./ \mathrm{m} \ell\right)$ da cepa AS-28, via oral, representando a principal via de infecção em cães jovens. Com uso de sonda naso-esofágica, os oocistos obtidos de fezes de gatos previamente inoculados com cisto destas cepas. O terceiro grupo de cães foi inoculado com $5 \mathrm{~m} \ell$ (1.9x $10^{7}$ taquizoítos/m $\ell$ ) da cepa $\mathrm{RH}$, divididos na seguinte proporção: $2,5 \mathrm{~m} \ell$ via intraperitoneal e $2,5 \mathrm{~m} \ell$ intravenosa concomitantemente. Isso foi feito para evitar o óbito dos animais, visto que esta cepa é altamente patogênica e não formadora de cisto ou oocisto, obtidos de lavado peritoneal de camundongos previamente inoculados. O quarto grupo, controle, foi inoculado com solução salina $0,85 \%$ estéril, sendo um via oral e o outro via intraperitoneal e intravenosa.

Os exames clínicos foram realizados duas vezes ao dia, durante todo o período experimental (30 dias). Compreendiam avaliações clínicas, neurológicas e oftalmológicas capazes de detectar alterações inerentes à evolução clínica da toxoplasmose. Todos os animais foram submetidos ao exame oftalmológico, pré e pós-inoculação, semanalmente, incluindo exame de fundo de olho, logo após a dilatação da pupila com a instilação de tropicamida (Midriacyl®). Os cães que apresentaram alterações na oftalmoscopia direta foram submetidos à oftalmoscopia indireta e tiveram os olhos fotomicrografados. Os soros dos animais na pré e na pós- inoculação, obtidos a cada três ou quatro dias durante todo o experimento, foram submetidos à sorologia para pesquisa de anticorpos IgG anti- $\boldsymbol{T}$. gondii pela RIFI, segundo CAMARGO (1964). Ao final do experimento, 30 dias após inoculação, os animais foram submetidos a eutanásia e a exames de necropsia. Após avaliação de ambos os olhos, retina e anexos oculares foram colhidos para exames histopatológicos, bem como fragmentos de baço, cérebro, coração, fígado, linfonodos, medula espinhal, músculo, pulmão e rins. 
Fragmentos do coração, linfonodos, medula espinhal, musculatura esquelética, pulmão e rins foram submetidos à digestão péptica (JACOBS \& MELTON, 1957). Os demais órgãos dos cães foram macerados, ressuspensos em salina estéril a $0,85 \%$ e adicionados de 2000UI de penicilina e $200 \mathrm{mg} / \mathrm{m} \ell$ de estreptomicina. Esses produtos assim obtidos foram inoculados, via intraperitonial, em grupos de três camundongos e observados durante 35 dias (DUBEY et al.,1995).

\section{RESULTADOS}

Durante os dias que antecederam a inoculação, nenhum sinal clínico foi observado nos cães. Após a inoculação, o único sinal clínico observado foi aumento dos linfonodos submandibulares em todos os cães inoculados com as três cepas, sendo que os animais controle não apresentaram qualquer alteração durante todo o período do experimento.

Os resultados destes exames, obtidos através da RIFI, foram considerados positivos quando apresentaram títulos maiores ou iguais a 1:16 (ISHIZUKA, et al., 1974/1981; GERMANO et al., 1985; GUIMARÃES et al., 1992), os quais se encontram na tabela 1. Anticorpos IgG anti- $\boldsymbol{T}$. gondii foram detectados nos cães 1, 2, 8 e 12 a partir do $4^{\circ}$ dia pós-infecção; nos cães 5,6 e 7 no $7^{\circ}$ D P I (dia após a inoculação), e no cão 3 a partir do $11^{\circ} \mathrm{D}$ P I. Os maiores níveis de anticorpos séricos foram obtidos somente após o 14ํㅡ DPI, com títulos máximos de 1:4096 nos cães inoculados com a cepa RH. Não foram encontrados sororreagentes nos animais do grupo controle (Tabela 1).

Tabela 1 - Títulos de anticorpos IgG anti-T. gondii à Reação de ImunofIuorescência Indireta em cães jovens inoculados experimentalmente com três cepas do parasito.

\begin{tabular}{lllllllllll}
\hline Cão & Cepa & \multicolumn{10}{c}{ Dias após a inoculação (D.P.I) } \\
\hline & & 0 & 4 & 7 & 11 & 14 & 18 & 21 & 25 & 28 \\
1 & AS-28 & 00 & 16 & 16 & 16 & 64 & 64 & 64 & 16 & 16 \\
2 & AS-28 & 00 & 16 & 16 & 16 & 16 & 16 & 16 & 16 & 64 \\
3 & AS-28 & 00 & 00 & 00 & 16 & 256 & 64 & 64 & 16 & 16 \\
4 & $\mathrm{P}$ & 00 & 00 & 16 & 16 & 64 & 64 & 16 & 16 & 16 \\
5 & $\mathrm{P}$ & 00 & 00 & 16 & 16 & 64 & 64 & 64 & 256 & 256 \\
6 & $\mathrm{P}$ & 00 & 00 & 16 & 16 & 16 & 64 & 16 & 16 & 64 \\
7 & $\mathrm{RH}$ & 00 & 00 & 64 & 1024 & 1024 & 4096 & 1024 & 1024 & 1024 \\
8 & $\mathrm{RH}$ & 00 & 256 & 1024 & 4096 & 1024 & 1024 & 1024 & 1024 & 1024 \\
9 & $\mathrm{RH}$ & 00 & 64 & 1024 & 1024 & 1024 & 256 & 256 & 256 & 1024 \\
10 & Controle & 00 & 00 & 00 & 00 & 00 & 00 & 00 & 00 & 00 \\
11 & Controle & 00 & 00 & 00 & 00 & 00 & 00 & 00 & 00 & 00 \\
12 & Controle & 00 & 00 & 00 & 00 & 00 & 00 & 00 & 00 & 00 \\
\hline
\end{tabular}

Positivo título maior ou igual a 16.
O exame de fundo de olho mostrou alterações com áreas de hiperrefletividade, na $\$$ semana, nos cães 8 e 9 inoculados com a cepa RH e, na $4^{\mathrm{a}}$ semana, nos cães $1 \mathrm{e} 2$, inoculados com a cepa AS 28; nos cães 4 e 5 com a $P$, e no cão 7 inoculado com a RH . Vitreíte foi dbservada no cão 7 na $3^{\text {a }}$ semana pós-inoculação. Os cães do grupo controle não apresentaram qualquer alteração. Dos sete animais que apresentaram alterações, foram selecionados três, que apresentavam lesões mais características, para a fotomicrografia. O cão 2 apresentou papiledema e edema perivascular no olho direito e papiledema exsudato peripapilar no esquerdo (Figura 1). O cão 8 apresentou papiledema, exsudato peripapilar e áreas de hiperrefletividade no olho direito (Figura 2) e não foi fotomicrografado olho esquerdo. O cão 9 apresentou papiledema e exsudato perivascular inferior no olho direito, e papiledema e tapeto hipopigmentado na porção anterior do olho esquerdo (Figura 3). As lesões histopatológicas de olho estão demonstradas na tabela 2.

Em pelo menos três amostras dos diferentes tecidos colhidos da cada cão, foi possível isolar o parasita, sendo que do baço foi isolado em sete cães; da retina, rins e cérebro em seis e da medula espinhal, músculo esquelético e do coração em cinco cães. Nas amostras colhidas do grupo controle, não foi isolado o parasita (Tabela 3).

\section{DISCUSSÃO}

Todos os cães inoculados responderam ao estímulo imunogênico. Foram produzidos anticorpos anti-T. gondii, já no \& DPI, em quatro animais, e alcançados títulos máximos por volta do $14^{\circ}$ após a inoculação (Tabela 1). Os resultados reforçam a característica antigênica particular de cada cepa do T.gondii nos hospedeiros (DRESSEN, 1990; DUBEY et al., 1995). O fato de apresentarem maiores títulos de anticorpos não significa maiores lesões, oriundas da presença de cistos, demonstrados nos achados do isolamento nos tecidos (Tabela 2). Os exames de oftalmoscopia direta e a retinografia (Figuras 1, 2, 3) apresentaram resultados sem precedentes na literatura referente à toxoplasmose canina, principalmente pela precocidade e variabilidade das alterações observadas. Apenas FIALHO (1953), que observou alterações oculares em cães experimentalmente inoculados, e PIPER $\boldsymbol{e} t$ al. (1970) descreveram retinite e coroidite e um cão adulto. 


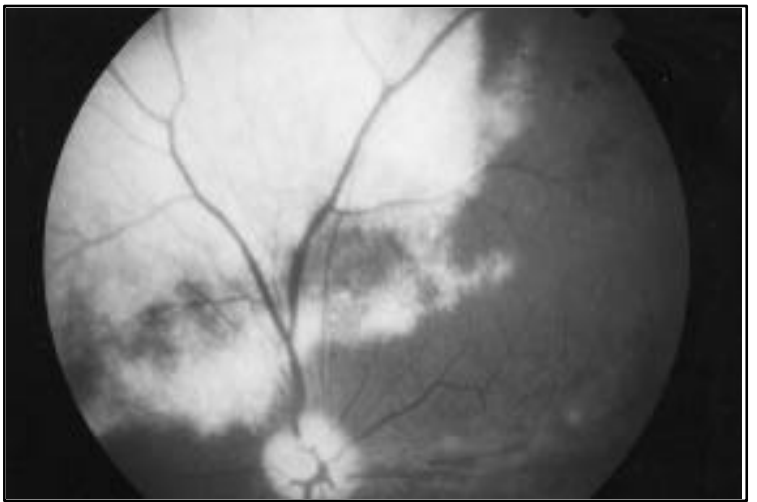

Figura 1 - Retinografia do Olho esquerdo do cão 2 inoculado, experimentalmente, com oocistos do Toxoplasma gondii, apresentando papiledema, exsudato peripapilar.

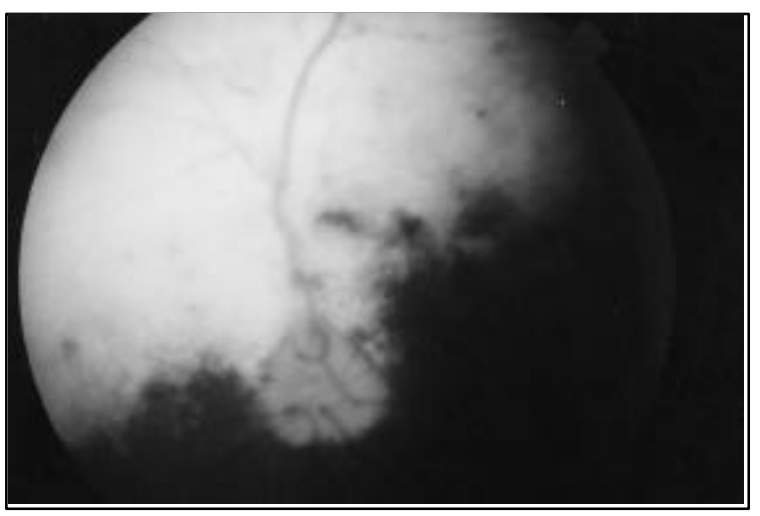

Figura 2 - Retinografia do olho direito do cão 8 inoculado, experimentalmente, com taquizoítas do Toxoplasma gondii, apresentando papiledema, exsudato peripapilar e áreas de hiperrefletividade.

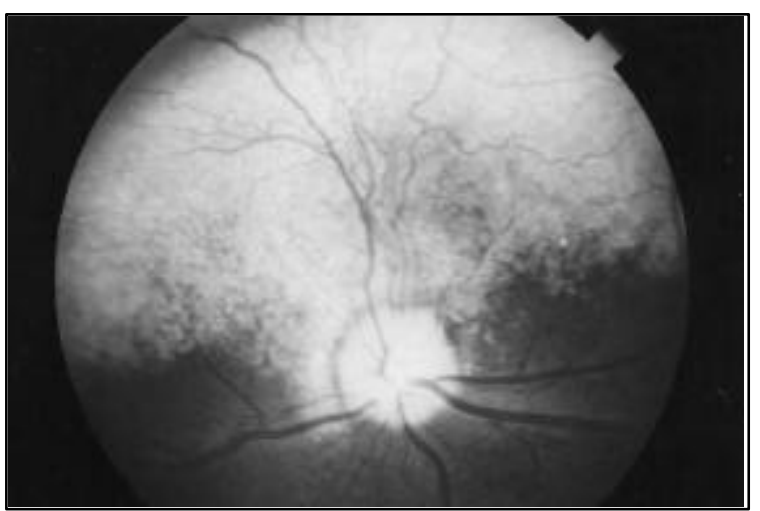

Figura 3 - Retinografia olho esquerdo do cão 9 inoculado, experimentalmente, com taquizoítas do Toxoplasma gondii, apresentando papiledema e tapeto hipopigmentado na porção anterior.

No entanto, na toxoplasmose humana há inúmeras descrições de alterações semelhantes aos observados nos cães, como as de DRESSEN (1990), HOLLAND et al. (1995) e SILVEIRA (1997), que mostraram casos que vão desde simples edema de papila até necrose ocular gravíssima e evolução para cegueira .

As alterações observadas clinicamente e confirmadas pela retinografia vem de encontro aos achados do isolamento do parasito em todos os tecidos pesquisados e na retina, quando inoculado em camundongos (Tabela 3), em que seis cães apresentaram o parasito no olho, principalmente nos inoculados com cepas formadoras de cisto, como é o caso da AS-28 e P. A cepa RH, com características à resposta aguda e à não cronificação DUBEY et al. (1995), foi isolada em apenas um cão, provavelmente ainda na fase de parasitemia. Os dois outros cães, 7 e 8 , apesar do não isolamento na retina, apresentaram alterações no exame direto do fundo de olho. $\mathrm{O}$ cão 8 também apresentou alterações na retinografia. Esse achado é justificado pelo fato de o taquizoíto, na sua fase aguda ou invasiva, poder provocar lesões gravíssimas sem, no entanto, levar à cronificação ou ao isolamento do agente em tecidos.

Esse fato foi também demonstrado por PIPER et al. (1970), que realizaram um estudo profundo da toxoplasmose oular em cães, gatos, ovinos e bovinos inoculados experimentalmente. Esses autores afirmaram que a evolução do quadro é mais evidente quando observado tardiamente.

Os resultados dos exames histopatológicos mostraram alterações tais como: edema subretiniano, infiltrado inflamatório focal, células mononucleares no espaço subretinal e coroidite, demonstrando a evolução do quadro, próprio da infecção por $\boldsymbol{T}$. gondii (Tabela 2), permitindo, assim, reforçar as observações no exame clínico e isolamento da retina do $\boldsymbol{T}$. gondii. Confirmam esses resultados os cães 2, 4 e 5, que apresentaram alterações significativas nas três avaliações. Os demais cães confirmam a toxoplasmose ocular por pelo menos duas avaliações, o que valida os achados.

Da mesma forma, SILVEIRA (1997), estudando a toxoplasmose ocular adquirida em crianças e adultos, afirma que as manifestações clínicas são tardias, mesmo que tenha sido adquirida precocemente. GIRALDI (1999) reforça essa afirmação, quando encontrou $35 \%$ de crianças que freqüentavam crechee escolas de $1^{\circ}$ grau com sorologia positiva para $\boldsymbol{T}$. gondii e nenhum caso de alteração ocular, quer pelo exame direto quer pela fundoscopia.

Apesar de o período experimental ter sido de apenas 30 dias, foi possível demonstrar as alterações oftálmicas provocadas pelo $T$. gondii em cães, com achados importantes para a interpretação do quadro. Sugerem-se, ainda, estudos mais prolongados para acomp anhar a evolução do quadro. 
Tabela 2 - Achado histopatológico do globo ocular de cães jovens inoculados, experimentalmente, com três cepas de Toxoplasma gondii, em Londrina, PR.

\begin{tabular}{|c|c|c|c|c|c|c|c|c|c|c|c|c|}
\hline \multirow[t]{2}{*}{ Lesões } & \multicolumn{12}{|c|}{ Cães } \\
\hline & 1 & 2 & 3 & 4 & 5 & 6 & 7 & 8 & 9 & 10 & 11 & 12 \\
\hline $\begin{array}{l}\text { Córnea, região ao ângulo da íris e estroma conjuntivo com infiltra- } \\
\text { do MN, em camada subepitelial e na região da coróide e congestão vascular. }\end{array}$ & & & & & + & & & & & & & \\
\hline $\begin{array}{l}\text { Vasos de retina com espessamento de parede e poucas células mo- } \\
\text { nonucleares. }\end{array}$ & & & & & & & & & & & & \\
\hline $\begin{array}{l}\text { Córnea, região ao ângulo da íris e estroma conjuntivo com infiltrado } \\
\text { MN, em camada subepitelial e na região da coróide e congestão vascular. }\end{array}$ & & & + & & & & & & & & & \\
\hline $\begin{array}{l}\text { Prolongamento de células fotorreceptoras de maneira espessada, } \\
\text { dando um aspecto de pregue amento. }\end{array}$ & & + & + & + & & & & & & & & \\
\hline Edema sub-retiniano e presença de líquido de coloração eosinofíli- & & + & & & + & & & & & & & \\
\hline $\begin{array}{l}\text { Congestão vascular de média intensidade na região da coróide, es- } \\
\text { clerótica e em glia da região de células ganglionares. }\end{array}$ & & & & & + & & + & & & & & \\
\hline $\begin{array}{l}\text { Congestão vascular com área focal de proliferação glial e presença } \\
\text { de raros elementos mononucleares. }\end{array}$ & & & & & & & & + & & & & \\
\hline Distribuição discreta de elementos celulares ganglionares. & + & + & + & + & + & + & + & + & + & & & \\
\hline
\end{tabular}

Grupo: 1, 2, 3, - cepa AS 28

Grupo: 4, 5, 6- cepa P.

Grupo: 7, 8, 9 - cepa RH.

Grupo: 10,11,12 - controle.

Tabela 3 - Isolamento de Toxoplasma gondii em grupo de três camundongos inoculados com amostras de órgãos, oriundos de cães jovens inoculados, experimentalmente, com três cepas do parasito.

\begin{tabular}{|c|c|c|c|c|c|c|c|c|c|c|c|}
\hline $\begin{array}{l}\text { Número de } \\
\text { cães/cepas }\end{array}$ & Pulmão & Coração & Músculo & Rim & Retina & Fígado & Baço & Linfonodo & Cérebro & Medula espinal & Total de positivos \\
\hline 01/As28 & Ps & Ps & - & Ps & Ps & Ps & Ps & Ps & $\mathrm{Pt}$ & Ps & 09 \\
\hline $02 / A s 28$ & - & $\mathrm{Ps} / \mathrm{Pc}$ & $\mathrm{Ps} / \mathrm{Pt}$ & Ps & Ps & Ps & - & Ps & Ps & - & 07 \\
\hline 03/As28 & - & Ps & Ps & Ps & Ps & Ps & Ps & Ps & Ps & Ps & 09 \\
\hline 04/P & - & $\mathrm{Pc}$ & $\mathrm{Ps} / \mathrm{Pc}$ & Ps & $\mathrm{Ps} / \mathrm{Pc}$ & - & Ps & Ps & $\mathrm{Ps} / \mathrm{Pc}$ & - & 07 \\
\hline $05 / \mathrm{P}$ & $\mathrm{Ps} / \mathrm{Pc}$ & Ps & - & - & $\mathrm{Ps} / \mathrm{Pc}$ & $\mathrm{Pc}$ & $\mathrm{Pc}$ & - & - & - & 05 \\
\hline $06 / \mathrm{P}$ & Ps & - & - & Ps & - & - & - & - & - & $\mathrm{Pc}$ & 03 \\
\hline $07 / \mathrm{RH}$ & - & - & - & Ps & - & Ps & Ps & - & - & Ps & 04 \\
\hline 08/RH & - & - & $\mathrm{Pt}$ & - & - & - & $\mathrm{Ps} / \mathrm{Pt}$ & - & $\mathrm{Pt}$ & Ps & 04 \\
\hline 09/RH & - & - & Ps & - & Ps & - & $\mathrm{Pc}$ & - & $\mathrm{Pt}$ & - & 04 \\
\hline 10/Cont & - & - & - & - & - & - & - & - & - & - & 00 \\
\hline $11 /$ Cont & - & - & - & - & - & - & - & - & - & - & 00 \\
\hline 12/Cont & - & - & - & - & - & - & - & - & - & - & 00 \\
\hline Total & 03 & 05 & 05 & 06 & 06 & 05 & 07 & 04 & 06 & 05 & 52 \\
\hline
\end{tabular}

-: negativo

Ps: positiva na sorologia pela RIFI para Toxoplasmose $(=1: 64)$ em camundongos inoculados com órgãos dos cães.

Pt: presença de taquizó́tos de $\boldsymbol{T}$. gondii no exsudato de camundongos inoculados com órgãos dos cães.

Pc: presença de cistos de $\boldsymbol{T}$. gondii nos encéfalos de camundongos inoculados com órgãos dos cães.

\section{CONCLUSÃO}

Todos os cães responderam ao estímulo antigênico, produzindo anticorpos anti- $\boldsymbol{T}$. gondii. Apresentaram alterações oftálmicas significativas, observadas, já na terceira semana pós-inoculação, através de microscopia direta e confirmadas na retinografia.

\section{REFERÊNCIAS BIBLIOGRÁFICAS}

ABREU, M.T., BELFORT Jr., R., ORÉFICE, F. Toxoplasmose ocular. In: ORÉFICE, F., BELFORT Jr., R. Uveítes. São Paulo : Roca, 1987. p.211-330.

AMATO NETO, V.; MEDEIROS, E.A.S. de, LEVI, et al. Toxoplasmose. São Paulo : Sarvier, 1995. p.250.

Ciência Rural, v. 32, n. 5, 2002. 
BANETH, G., SHKKAP, V., SAVITSKY, I. et al. The prevalence of antibodies to Toxoplasma gondii in dogs in Israel. Israel J Vet Med, v.51, n.1, p.31-33, 1996.

CAMARGO, M.E. Improved technique of indirect immunuofluorescence for serological diagnosis of toxoplasmosis. Rev Inst Med Trop, v.6, n.3, p.117-118, 1964.

DRESSEN, D.W. Toxoplasma gondii. J Am Vet Med Assoc, v.196, n.2, p.274-276, 1990.

DUBEY, J.P. Toxoplasma, Neospora, Sarcocystis and other tissue cyst-forming of human and animals. In: KRIER, J.P. (coord). Parasitic protozoa. 2.ed. San Diego : Academic 1993. p.157.

DUBEY, J.P., WEIGEL, R.M., SIEGEL, A.M. et al. Sources and reservoirs of Toxoplasma gondii infection on 47 swine farms in Illinois. J Parasitol, v.81, n.5, p.723-729, 1995.

FIALHO, S.A. Toxoplasmose ocular: contribuição ao estudo clínico e experimental. Rio de Janeiro, 1953. 139p. Tese (Doutorado em Medicina) - Faculdade Nacional de Medicina da Universidade do Brasil, 1953.

GIRALDI, N. Estudo da Toxoplasmose em escolares do 1ํㅡㅁ grau do Município de Rolândia. Londrina - Paraná. 1999. 64p. Dissertação (Mestrado em Sanidade Animal) - Curso de Pós-graduação em Sanidade Animal, Universidade Estadual de Londrina, 1999.

GERMANO, P.M.L., ERBOLATO, E.B., ISHIZUKA, M.M. Estudo sorológico da toxoplasmose canina, pela prova de imunuofluorescência indireta, na cidade de Campinas, 1981. Rev Fac Med Vet, v.22, p.53-58, 1985

GUIMARÃES, A.M.; RIBEIRO, M.F.B.; LIMA, J.D. Freqüência de anticorpos anti-Toxoplasma gondii em cães de Belo Horizonte, MG. Arq Bras Med Vet Zootec, v.44, n.1, p.67-68, 1992.

ISHIZUKA, M.M.; MIGUEL, O.; BROGLIATO, D.F. Prevalência de Anticorpos anti-Toxoplasma gondii em soro de cães no município de São Paulo. Rev Fac Med Vet Zootec, v.11, p.115-125, 1974.

ISHIZUKA, M.M., MIGUEL, O., BROGLIATO, D.F. Incidência de infecção por Toxoplasma gondii em cães do município de São Paulo. Rev Fac Med Vet Zootec, v.18, p.161-165, 1981.

JACOBS, L., MELTON, M.L. A procedure for testing meat samples for Toxoplasma gondii with preliminar results of a survey of pork and beef sample. J Parasitol,v.42, n.2, p.3839, 1957.

HOLLAND, G.N., WILHEMUS, K.R. Ocular infect immun. St. Louis : Mosby, 1995. p.1183-223.

PIPER, R.C., CLOE, C.R., SHAFFUCK, J.A. Natural and experimental ocular toxoplasmosis in animals. Am J Opht, v.69, p.662-668, 1970.

SILVEIRA, C.A.M. Estudo da toxoplasmose ocular na região de Erechim - RS. São Paulo, 1997. 204p. Tese (Doutorado em Medicina) - Escola Paulista de Medicina, 1997. 